\title{
Human genetic variants associated with COVID-19 severity are enriched in immune and epithelium regulatory networks
}

Zhanying Feng ${ }^{1,2}$, Xianwen Ren $^{3}$, Zhana Duren ${ }^{4}$, and Yong Wang ${ }^{1,2,5,6^{*}}$

${ }^{1}$ CEMS, NCMIS, HCMS, MDIS, Academy of Mathematics and Systems Science, Chinese Academy of Sciences, Beijing 100190, China

${ }^{2}$ School of Mathematics, University of Chinese Academy of Sciences, Chinese Academy of Sciences, Beijing, China ${ }^{3}$ School of Life Sciences, Peking University, Beijing 100871, China

${ }^{4}$ Center for Human Genetics and Department of Genetics and Biochemistry, Clemson University, Greenwood, SC, 29646, USA

${ }^{5}$ Center for Excellence in Animal Evolution and Genetics, Chinese Academy of Sciences, Kunming 650223, China ${ }^{6}$ Key Laboratory of Systems Biology, Hangzhou Institute for Advanced Study, University of Chinese Academy of Sciences, Chinese Academy of Sciences, Hangzhou, 330106, China

\begin{abstract}
Human genetic variants can influence the severity of infection with SARS-COV-2. Several genomewide association studies (GWAS) have been conducted to identify human risk loci that may be involved with COVID-19 severity. However, candidate genes were investigated in the genomic proximity of each locus without considering their functional cellular contexts. Here, we compiled regulatory networks of 77 human contexts to interpret these risk loci by revealing their relevant contexts and associated transcript factors (TF), regulatory elements (REs), and target genes (TGs). 21 human contexts were identified to be associated with COVID-19 severity and grouped into two categories: immune cells and epithelium cells. We further investigated the risk loci in regulatory network of immune cells, epithelium cells and their crosstalk. Two genomic clusters, chemokine receptors cluster and OAS cluster showed the strongest association with COVID-19 severity in the context specific regulatory networks.
\end{abstract}

\section{Introduction}

Coronavirus disease (COVID-19) is an infectious disease caused by the SARS-CoV-2 virus. Most people infected with the virus will experience mild to moderate respiratory illness and recover without requiring special treatment. However, some will become seriously ill and require medical attention. Scientists from all over the world have made great efforts to understand the genetic mechanism of COVID-19 severity, which may lead to efficient prevention stratagem and effective cure. Genome-wide association analysis (GWAS) has been largely used to find human genetic variants associated with some phenotypes. Recently, applying GWAS to phenotypes of COVID-19, such as infection and severe respiratory symptoms, has helped us identify some risk loci associated with COVID-19. For example, the "The Severe COVID-19 GWAS Group" conducted a GWAS involving 1,980 patients with severe COVID-19 symptoms and detected SNP rs11385942 at locus 3 p21.31 and rs657152 at locus 9q34.2 ${ }^{1}$. Erola P. C. et al. used GWAS to study 2,244 critically ill COVID-19 patients from 208 UK intensive care units (ICUs) and found rs10735079 on 12q24.13 in a gene cluster encoding antiviral restriction enzyme activators (OAS1, OAS2, OAS3), rs2109069 on 19p13.2 near the gene TYK2, rs2109069 on 19p13.3 within DPP9, and rs2236757 on 21q22.1 in 
the interferon receptor gene IFNAR2 2. "The COVID-19 Host Genetics Initiative" conducted several GWAS, including "very severe respiratory confirmed covid" and "hospitalized covid", and also found many potential loci". These identified risk genetic variants allow us to further understand the underlying mechanism of COVID-19 severity.

Interpreting these COVID-19 associated genetic variants remains challenging since many studies revealed that most genetic variants were located in the non-coding regulatory region with high linkage disequilibrium ${ }^{4-6}$ and in time- and space-specific comtexts ${ }^{7}$. This implies that our task is to elucidate these genetic variants in the proper cellular contexts. Several works have been done to uncover the regulatory mechanism of genetic variants. For example, FUMA ${ }^{8}$ and GREAT ${ }^{9}$ used the routine way to link SNPs to the nearby genes. $\mathrm{SMR}^{10}$ and Sherlock ${ }^{11}$ utilized the expression information from eQTL to find SNP involved regulation. However, these methods either ignored the cis-regulation or not fully utilized the regulatory network. Recently, we developed a novel method (PECA) to infer regulatory network with paired expression and chromatin accessibility data $^{12}$ and then extended to PECA2 to reconstruct regulatory network for one sample with paired expression and chromatin accessibility data ${ }^{13}$. The reconstructed regulatory networks have been successfully applied to reveal critical regulations for time course data ${ }^{14}$. To make the most use of the public available paired expression and chromatin accessibility data, such as ENCODE and ROADMAP, we used PECA2 to construct a regulatory network atlas of 77 human contexts, which served as a valuable resource for genetic variants interpretation in multi-cellular contexts.

Here, we utilized these 77 regulatory networks to interpret the SNPs of COVID-19 severity. We first found the relevant tissues of COVID-19 severity were categorized into two main cell types: immune cells and epithelium cells. Then in these two cell type categories, we illustrated the detailed SNP associated regulation: SNP located in regulatory elements (REs), their upstream transcription factors (TFs), and their downstream target genes (TGs). We found that two gene clusters (chemokine receptors and OAS cluster) were important TGs in COVID-19 associated regulatory networks and showed different regulation patterns in two cell type categories. Our COVID-19 severity associated regulatory network will be promising to serve as a valuable perspective to study COVID- 19 .

\section{Results}

\section{Human genetic variants of COVID-19 severity are enriched in immune and epithelium cells}

We first constructed a regulatory network atlas of human contexts. To do this, we collected paired expression and chromatin accessibility data of 76 human contexts, which included samples from all three germ layers, such as frontal cortex (ectoderm), primary T cells (mesoderm), and upper lobe of left lung (endoderm). With each sample's paired expression and chromatin accessibility data as input, we used PECA2 model $^{13}$ to construct regulatory network for each context (Methods). The basic unit of regulatory network was TF-REs-TG triplet and each triplet meant that TF bound on the REs and regulated the TG. We also included the regulatory network of cranial neural crest cells ${ }^{15}$ (CNCC), a migratory cell population in early human craniofacial development, into this regulatory network atlas. We applied these regulatory networks of 77 human contexts for interpretation of genetic variants of COVID-19 severity. 
A

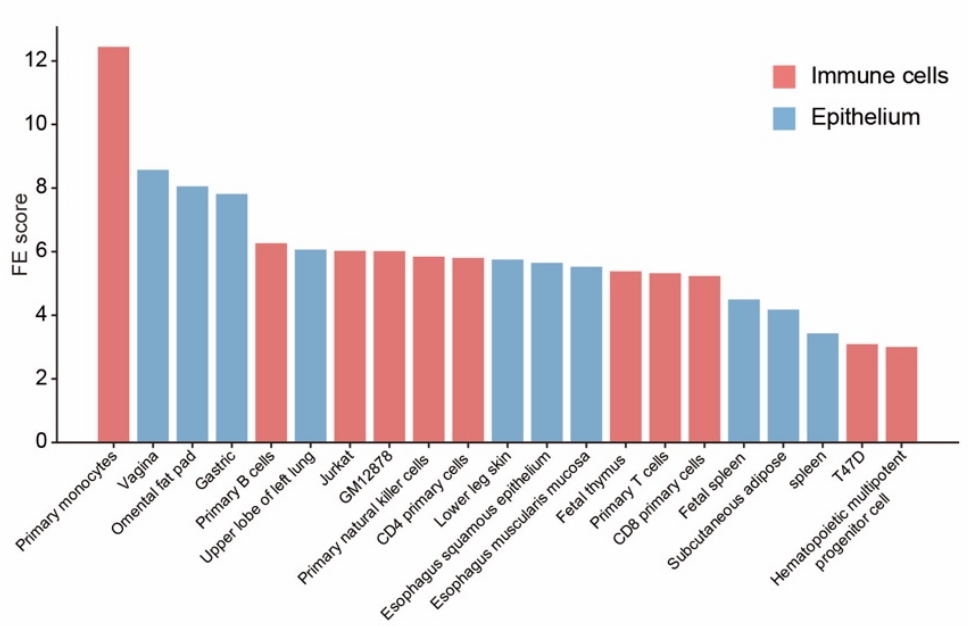

B Primary monocytes

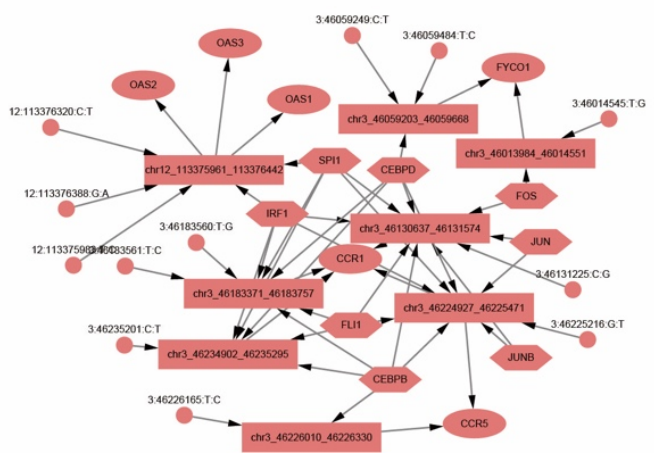

C Upper lobe of left lung

Figure 1. (A). 21 COVID-19 severity relevant contexts ranked by FE score. Red: immune cells. Blue: epithelium cells. (B). COVID-19 SNP associated regulatory of "fetal thymus", which is an immune cell type. (C). COVID-19 SNP associated regulatory of "upper lobe of left lung", which is an epithelium cell type.

We fetched the 542 SNPs with significant associations with "very severe respiratory confirmed COVID" ("A2_ALL" study in "The COVID-19 Host Genetics Initiatives"). To evaluate the relevance between SNPs and 77 human contexts, the fold enrichment (FE) score of these 542 SNPs in RE sets of 77 human contexts were calculated (Methods). We set the threshold of FE score to be 3.0 and found that 21 contexts were relevant to SNPs of COVID-19 severity, such as "primary monocytes" and "upper lobe of left lung" (Figure 1A). We found these 21 contexts could be classified into two categories. The first category was immune cells, including 11 cell types: "primary monocytes", “primary B cells", “Jurkat", "GM12878”, “primary natural killer cells", "CD4 primary cells", “fetal thymus”, “primary T cells", “CD8 primary Cells”, “T47D”, and "hematopoietic multipotent progenitor cell". The second category was marked by epithelium cells and consisted of 10 cell types: "vagina", “omental fat pad", "gastric", "upper lobe of left lung”, "lower leg skin", “esophagus squamous epithelium”, “esophagus muscularis mucosa”, "fetal spleen", "subcutaneous adipose tissue", and "spleen".

We further extracted sub-network that was associated with SNPs of COVID-19 severity in every COVID-19 severity relevant context (Methods). For example, "primary monocytes" was one of the immune cell types. And 12 SNPs were located in the 8 REs of regulatory network of "primary 
monocytes" (Figure 1B). These REs were predicted to regulate 9 TGs, such as CCR 1 and FYCO1. CCR1 played a key role in T-cell-mediated respiratory inflammation ${ }^{16}$. These REs were bound by immunity associated TFs, such as $C E B P B / D^{17-20}$, and $F L I 1^{21}$. On the other hand, "upper lobe of left lung" was a cell type of epithelium cells (Figure 1C) and 8 REs were associated with 14 SNPs of COVID-19 severity and regulated 5 TGs, such as CCRI and OAS1. Polymorphisms of OAS1 have been reported to affect susceptibility to a variety of viral diseases ${ }^{22,23}$. Some TFs bound and regulating these REs were linked to lung epithelium, such as $C E B P D^{24}$ and $E T S 2^{25}$.

In summary, we built a regulatory network atlas of 77 human contexts and used these regulatory networks to find relevant contexts to genetic variants of COVID-19 severity. Two categories of tissues were found to be associated with COVID-19 severity: the first was epithelium cells and may be involved with susceptibility to viral diseases; the other category was immune cells and possibly linked to severity after being infected with disease.

\section{Construction of SNP associated regulatory network of immune and epithelium cells}

To obtain more understanding about these two categories of cell types, we constructed COVID-19 severity associated regulatory networks in immune cells and epithelium cells respectively. Briefly, we pooled the SNPs associated networks of 11 immune cell types into a COVID-19 severity associated regulatory network of immune cells. Similarly, we pooled the SNPs associated regulatory network of 10 epithelium cell types into a COVID-19 severity associated regulatory network of epithelium cells (Methods).

In COVID-19 severity associated sub-regulatory network of immune cells, there were totally 17 TFs, 25 REs and 15 TGs that were associated with 38 SNPs (Figure 2A). This sub-regulatory network was highly associated with immune functions. For example, TCF3/7/12 were important upstream TFs in regulatory network of immune cells and played vital roles during T-cell development ${ }^{26,27}$. IRF 1/3/4 were critical TFs in the cellular differentiation of hematopoietic cells and in the regulation of gene expression in response to pathogen-derived danger signals ${ }^{28}$. CCR5 was downstream TGs in this sub-regulatory network and it encoded a protein on the surface of white blood cells that is involved in the immune system ${ }^{29}$. In COVID-19 severity associated regulatory network of epithelium cells, there were totally $16 \mathrm{TFs}, 24$ REs and 10 TGs that were associated with 42 SNPs (Figure 2B). This SNP associated regulatory network was also involved with the functions of epithelium cells. For example, ETS2, which was core TF in regulatory network of epithelium cells, could promote epithelial-to-mesenchymal transition in renal fibrosis ${ }^{25}$. ELF3 was also essential for mesenchymal to epithelial transition ${ }^{30} . N B E A L 2$ was a TG in epithelium regulatory network and the Nbeal2-deficient mice exhibited impaired development of functional granulation tissue due to severely reduced differentiation of myofibroblasts in the model of excisional skin wound repair ${ }^{31}$. These literature evidence showed that the constructed SNP associated regulatory networks were promising to reveal COVID-19 severity associated regulations in immune and epithelium cells.

We further validated our SNP associated regulatory network with eQTL dataset. Our SNP associated regulatory network gave the link between SNPs and genes by SNP's location in REs. For example, SNP "12:113364332:A:G" was located in RE "chr12:113364318-113364636" and 


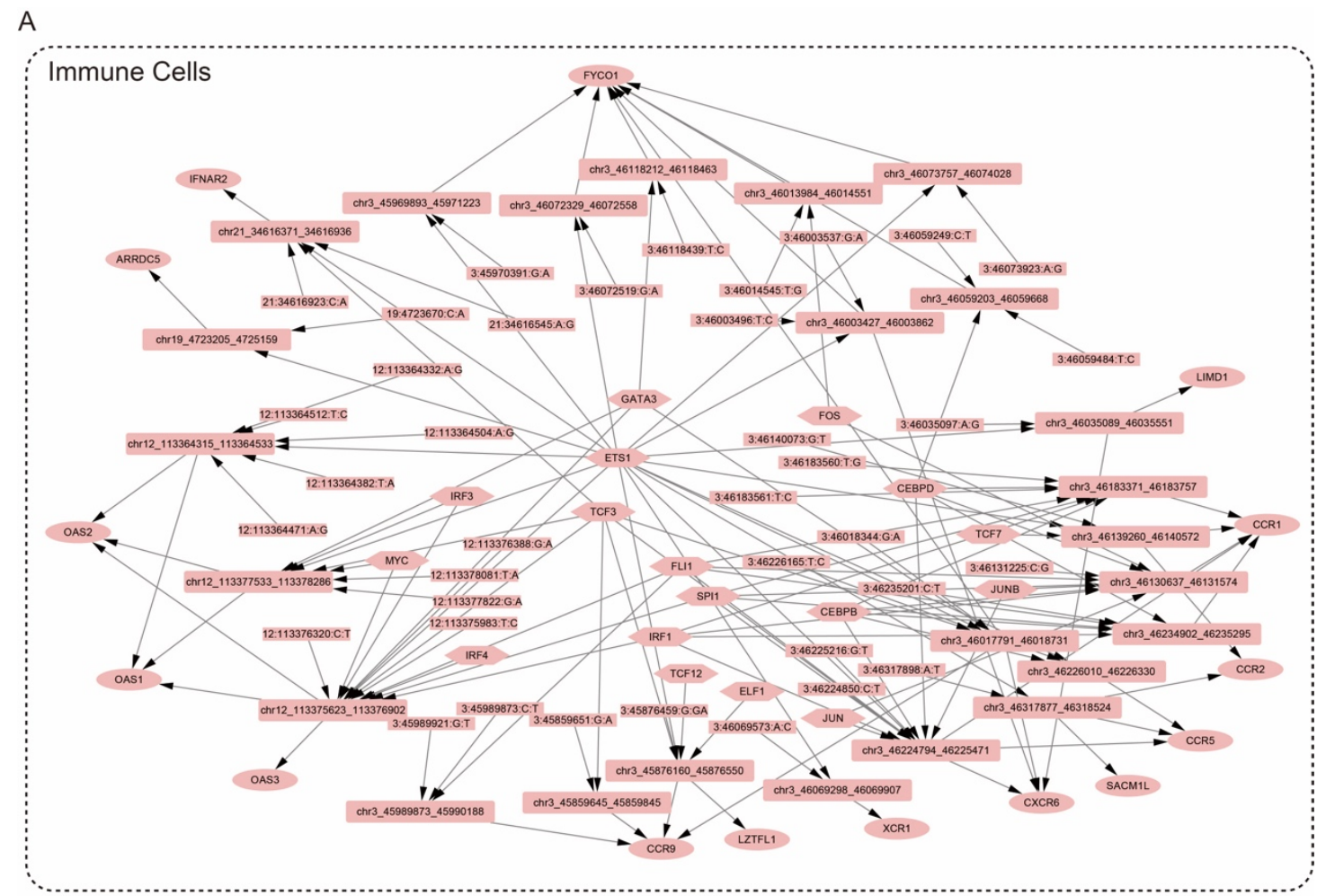

B

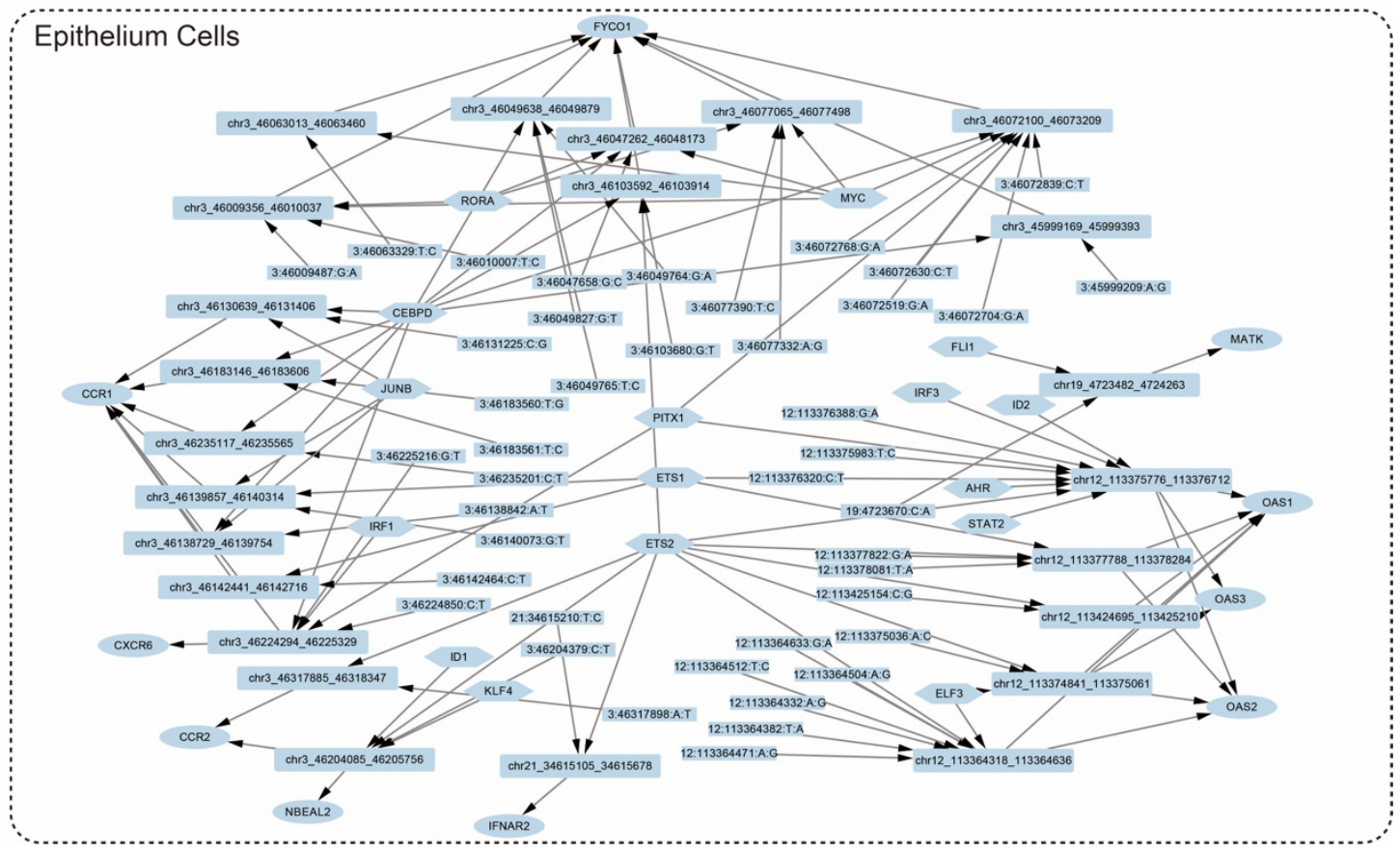

Figure 2. (A). COVID-19 severity associated regulatory network of immune cells. (B). COVID-19 severity associated regulatory network of epithelium cells.

"chr12:113364318-113364636" regulated OAS1, which gave the association between SNP “12:113364332:A:G” and gene OSA1. In this way, we totally obtained 91 SNP-TG association from immune and epithelium regulatory networks. Then we collected significant variants-gene association of 49 tissues from GTEx v8. We found that our SNP-TG associations were highly reproducible by eQTL variants-gene association. First of all, there were 78 (85.7\%) SNP-TG 
associations could be detected as an eQTL variant-gene association in at least one tissue. On the other hand, when we ranked the 49 tissues by their overlapping number of eQTL with our SNP-TG association, we found that the top ranked tissues were also immune and epithelium cell types (Figure 3). For example, "esophagus mucosa", which was one of epithelium cell types, ranked first by overlapping with SNP-TG association. There were some other tissues that were related to epithelium cells, such as "Cells Cultured fibroblasts", "Skin Not Sun Exposed Suprapubic", "Spleen", "Skin Sun Exposed Lower leg", "Lung”. And "Artery Tibial” and "Whole Blood” were parts of the blood, which were associated with immune functions. These results showed that our SNP-associated regulatory network could be evident by independent eQTL dataset.

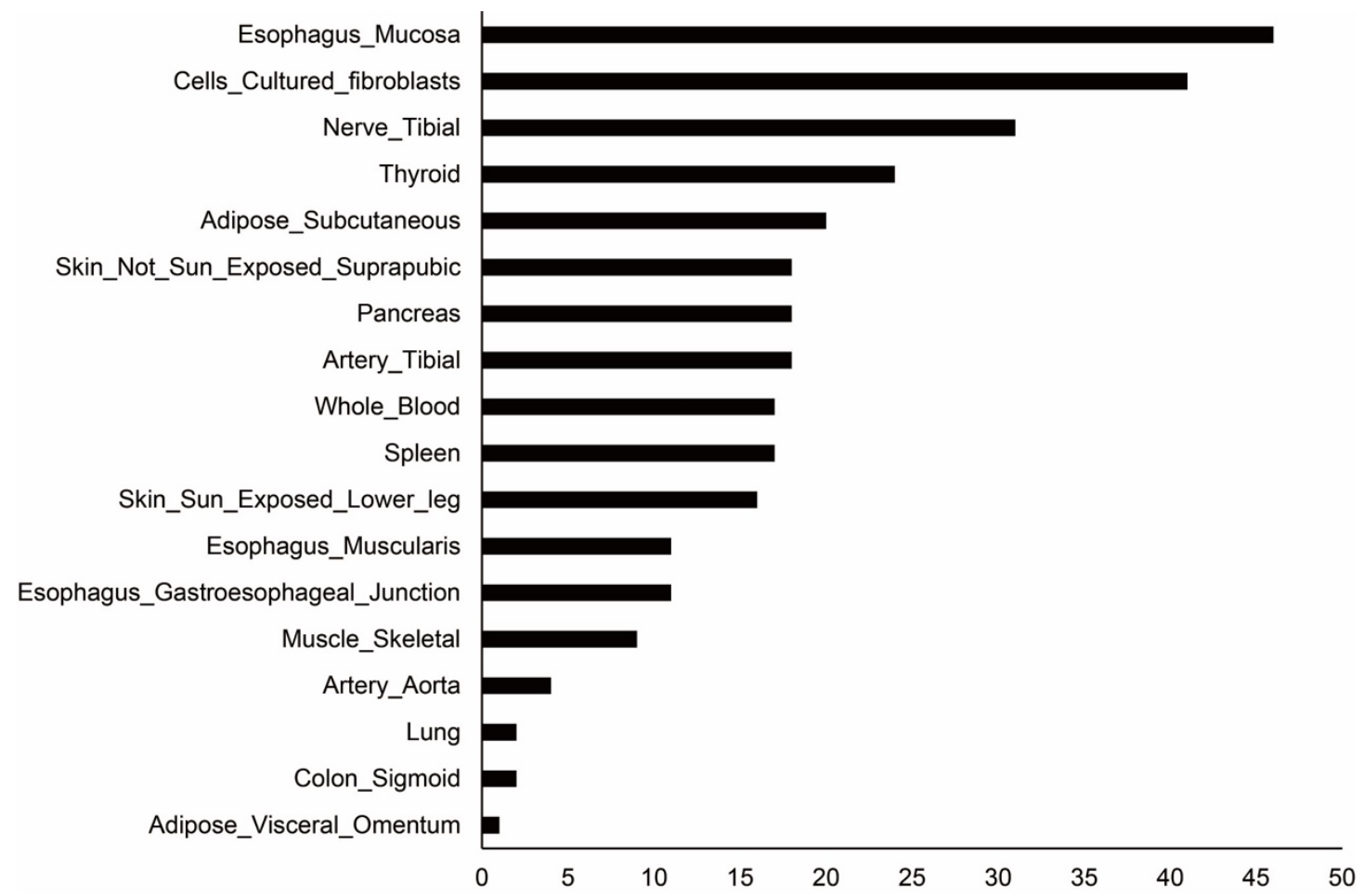

Figure 3. Overlapping of SNP-TG inferred from regulatory network with eQTL of 49 tissues in GTEx.

In summary, we have constructed COVID-19 severity associated regulatory network of immune cells and epithelium cells. These two regulatory networks were tightly linked to immune and epithelium functions and could be validated by eQTL of immune cells and epithelium tissues in GTEx.

\section{Associating two cell types' regulatory networks revealed regulatory structure of COVID- 19 severity}

After constructing and validating the regulatory networks of immune and epithelium cells, we next compared these two cell types to find conservation and divergence. We first compared the TFs, TGs, REs, and SNPs in the regulatory networks of immune and epithelium cells. For TFs, 7 TFs were shared by two cell types, such as ETSI and IRF1. There were 10 immune-specific TFs (such as TCF7 and ELF1) and 9 epithelium-specific TFs (such as ETS2 and KLF4). There were also 8 overlapped TGs (such as CCR1, FYCO1), 7 immune-specific TGs (such as CCR 5 and LZTFL1) and 2 epithelium-specific TGs (MATK and NBEAL2). For SNPs and REs, about half of them in immune regulatory network were shared by epithelium regulatory network (Figure 4). 
SNP

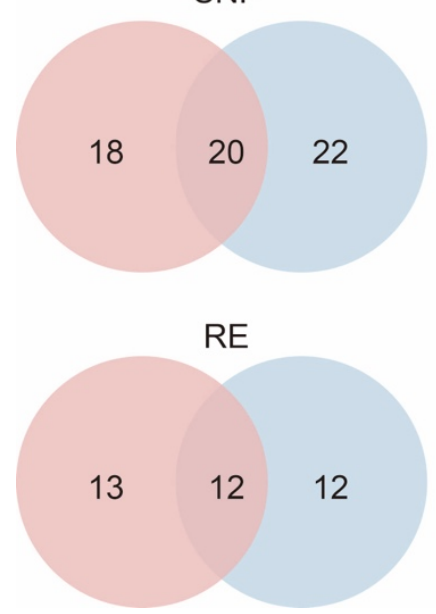

TF

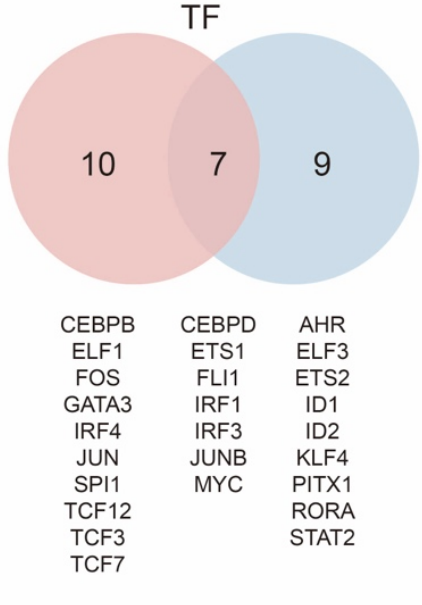

TG

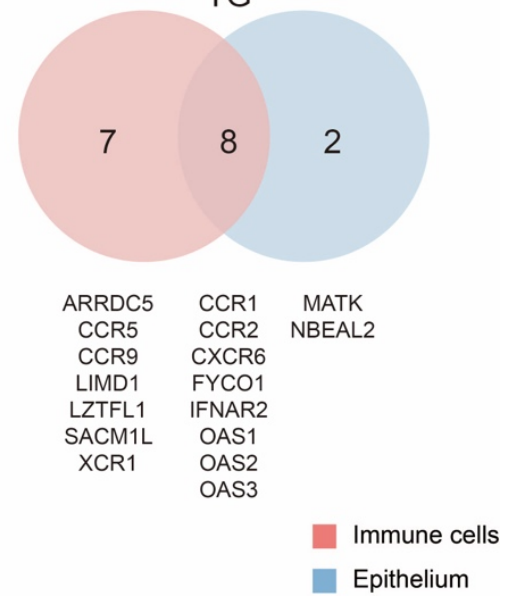

Figure 4. Overlapping of SNP, RE, TF, TG between immune regulatory network and epithelium regulatory network.

We found that the regulatory network could be clustered into four clusters according their location and this clustering was conserved between immune and epithelium cells (Figure 2A, B). The first cluster was on chromosome 3. This cluster was involved with regulation of CCR1/2/5/9, CXCR6, FYCO1, LIMD1, LZTFL1, SACMIL, XCR1 in immune cells and CCR1/2/5/9, CXCR6, NBEAL2 in epithelium cells. The second cluster was located in chromosome 12 and involved with regulation of $O A S 1 / 2 / 3$ in both immune and epithelium cells. The third cluster was in chromosome 19. In immune cells, this cluster was associated with $A R R D C 5$ and in epithelium cells, it was associated with MATK. The last cluster was in chromosome 21 and was linked to IFNAR2 in two cell types. These four regulatory clusters revealed four loci that may exert genetic influence on infection and severity of COVID-19.

In the regulatory network of two cell types, we found two classes of upstream TFs (Figure 2A, B). The first class of TFs regulated most of the four regulatory clusters. For example, in regulatory network of immune cells, ETS1, TCF3 and GATA3 were involved with at least three regulatory clusters. And for the epithelium cells, ETS1, ETS2, and PITX1 were classified into this class. Contrary to the broad regulation of the first class, the second class of TFs was only responsible for a small part of regulatory network. For example, in immune regulatory network, $C E B P D, C E B P B$, $T C F 7, F O S, J U N B, J U N, E L F 1$ were only regulate REs in chromosome 3 regulatory cluster. And $I R F 3, I R F 4$, and $M Y C$ only responsible for regulations in chromosome 12 regulatory cluster. In the epithelium regulatory network, CEBPD, JUNB, IRF1, ID1, RORA, MYC, and KLF4 were only involved with the regulatory of chromosome 3 regulatory cluster. ID2, IRF3, AHR, STAT2, ELF3 was solely associated with the chromosome 12 regulatory cluster.

Through the comparison of two cell types' regulatory networks, we found these two cell types shared many TFs, TGs, REs, and SNPs. The regulatory structure (four regulatory clusters based on location and two classes of upstream TFs) were conserved in two cell types. Despite the shared regulatory structure, there were also many cell-type-specific TFs, TGs, REs, and SNPs.

4. Chromosome 3 and 12 clusters showed distinct regulatory program in immune and 


\section{epithelium cells}

From the above analysis of regulatory structure, we noticed that there are two relatively denser clusters in immune and epithelium regulatory: chromosome 3 and chromosome 12 clusters, indicating that they may play an essential role in COVID-19 severity. Then we focused on these two regulatory clusters.

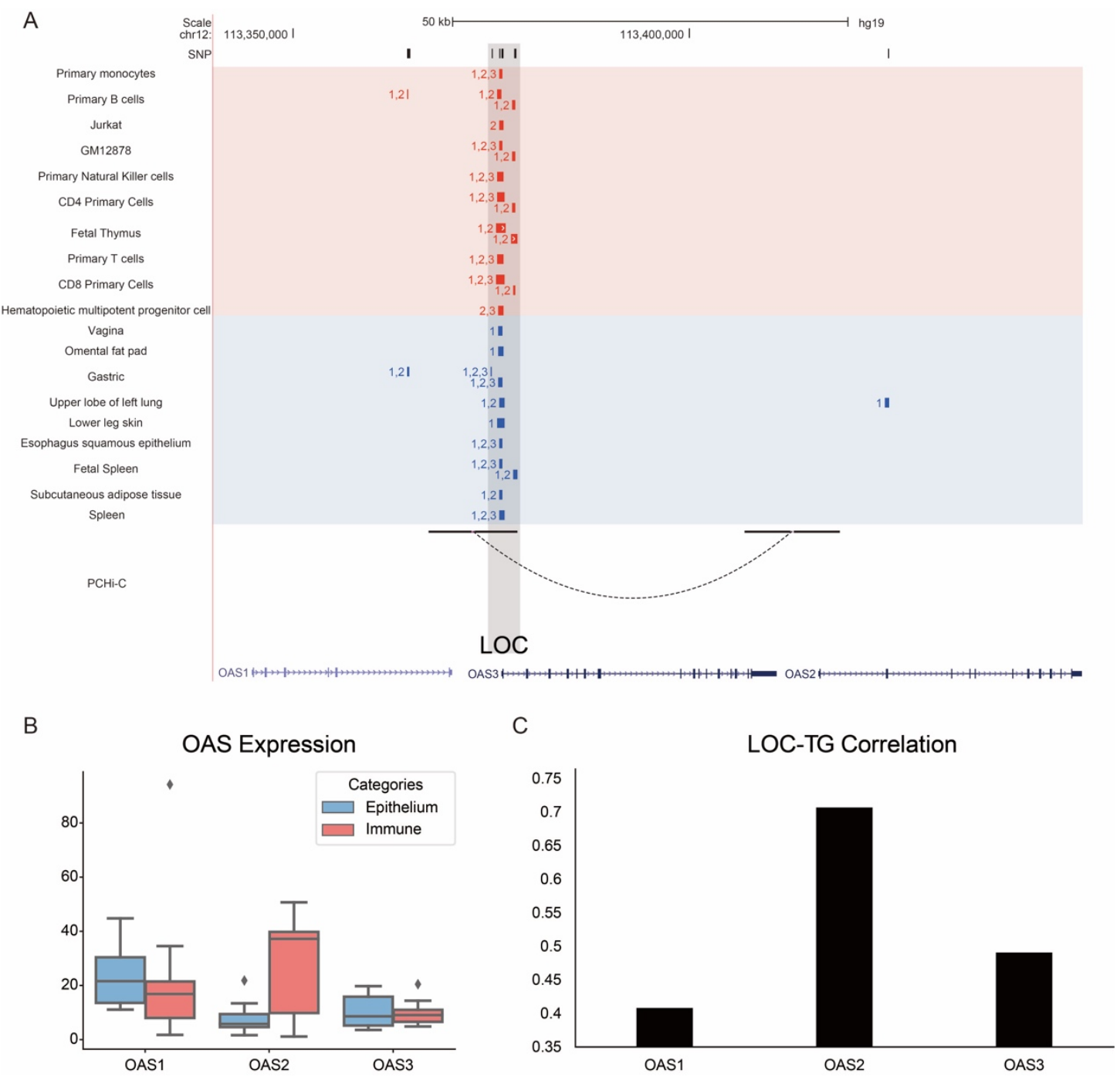

Figure 5. (A). SNPs, REs and PCHi-C loop associated with $O A S 1 / 2 / 3$. The RE tracks consisted of 11 immune cell types and 9 epithelium cell types. PCHi-C loops were from blood samples. The number labels around the RE were indicator of RE's target genes. (B). Expression of $O A S 1 / 2 / 3$ in immune and immune cells. (C). Correlations between accessibility of REs and expression of $O A S 1 / 2 / 3$.

For regulatory cluster in chromosome 12 , these regulatory programs were mainly involved with $O A S$ cluster $(O A S 1 / 2 / 3)$ in ten immune cell types and nine epithelium cell types. These $O A S$ cluster was important for immune and epithelium functions. For example, $O A S 1$ polymorphisms played potential roles in respiratory infection from human bronchial epithelial cells ${ }^{23}$. The $O A S 2$ protein is a well-known innate immune activated antiviral enzyme catalyzing synthesis of 2'-5'oligoadenylate for RNase L activation and inhibition of viral propagation ${ }^{32}$. We found that the REs of this cluster in different cell types were mainly distributed around the promoter of $O A S 3$ (Figure 5A) and there were many COVID-19 severity associated SNPs within these REs, which was 
consistent among immune and epithelium cell types. To add additional evidence of regulation in this area, we collected previously published promoter-capture Hi-C (PCHi-C) data of primary blood cell types $^{33}$. We used the PCHi-C interactions that have CHiCAGO scores $\geq 5$ in at least one analyzed cell type and found there was a loop between promoter of $O A S 3$ and promoter of OAS2. This observation induced a hypothesis that the REs around the promoter of OAS3 were cisregulatory elements of $O A S 2$ in immune cells. To validate this hypothesis, we first checked the REs regulating the three OAS genes in 10 immune cell types and found that all 15 REs were predicted to regulate $O A S 2,13$ REs were predicted to regulate $O A S 1$ and only 7 REs were predicted to regulate $O A S 3$, which revealed that more evidence supported that REs in this locus regulated $O A S 2$. Then we check the expression of three $O A S$ genes. We found $O A S 2$ 's expression was the highest among $O A S$ cluster in immune cell types (Figure 5B). We also computed the averaged correlation between the openness score of REs in OAS3's promoter and expression of three OAS genes among 148 samples of human (Table S1). We found that the accessibility of these REs was more correlated to expression of OAS2 (Figure 5C). In summary, these evidence of PCHi-C loop, regulatory network, expression data, and RE-TG correlation indicated that in immune cells, the REs around OAS3's promoter were more likely to regulate $O A S 2$.

For regulatory cluster in chromosome 3 , these regulatory programs were mainly involved with chemokine receptor (CCR1/2/5/9, CXCR6, and XCR1) in eight immune cell types and eight epithelium cell types. In literature, there were many reports that these chemokine receptors were important in the function of both immune and epithelium cells ${ }^{16,29}$. We found five main regulatory areas that were associated with SNPs and regulated these chemokine receptors (we name them as LOC1-LOC5, Figure 6A). Some of these REs' regulation was shared by two cell types. For example, LOC3 were predicted to regulate $C C R 1$ in both immune and epithelium cells. And there was also a PCHi-C loop between LOC3 and CCR1. The other LOCs' regulatory programs were different. For example, LOC1 and LOC2 were immune-specific REs and regulated $C C R 9$ and $C X C R 6$. While REs in LOC4 were shared by two cell types, they were predicted to regulate $C C R 5$ in immune cells but regulate $C C R 1$ in epithelium cells. LOC4 and CCR 5 were also contacted by a PCHi-C loop in blood cells. Similar to LOC4, LOC5 regulated CCR2/5 in immune cells but only regulate CCR2 in epithelium. The expression of these chemokine receptors supported the regulation above. $C C R 1 / 2 / 5 / 9$ and CXCR6 were relatively high expressed in immune cells but only CCR 1 and CCR 2 were expressed in epithelium cells (Figure 6B). We also checked the RE-TG correlation and found the above regulations were also supported. LOC1 showed the highest correlation with CCR9. LOC2 were more correlated with CXCR6 and CCR9. LOC3 was most associated with CCR1. LOC4 showed higher correlation with CCR1 and CCR5. And LOC5 was correlated with CCR5 (Figure 6C).

Taken together, we found some shared and distinct regulatory programs in immune and epithelium by detailed study of two main COVID-19 severity associated regulatory cluster on chromosome 3 and 12. These conserved and divergent regulations may give biological insights about how virus infected human through epithelium cells and how human defensed SARS-Cov-2 with our immune system. 


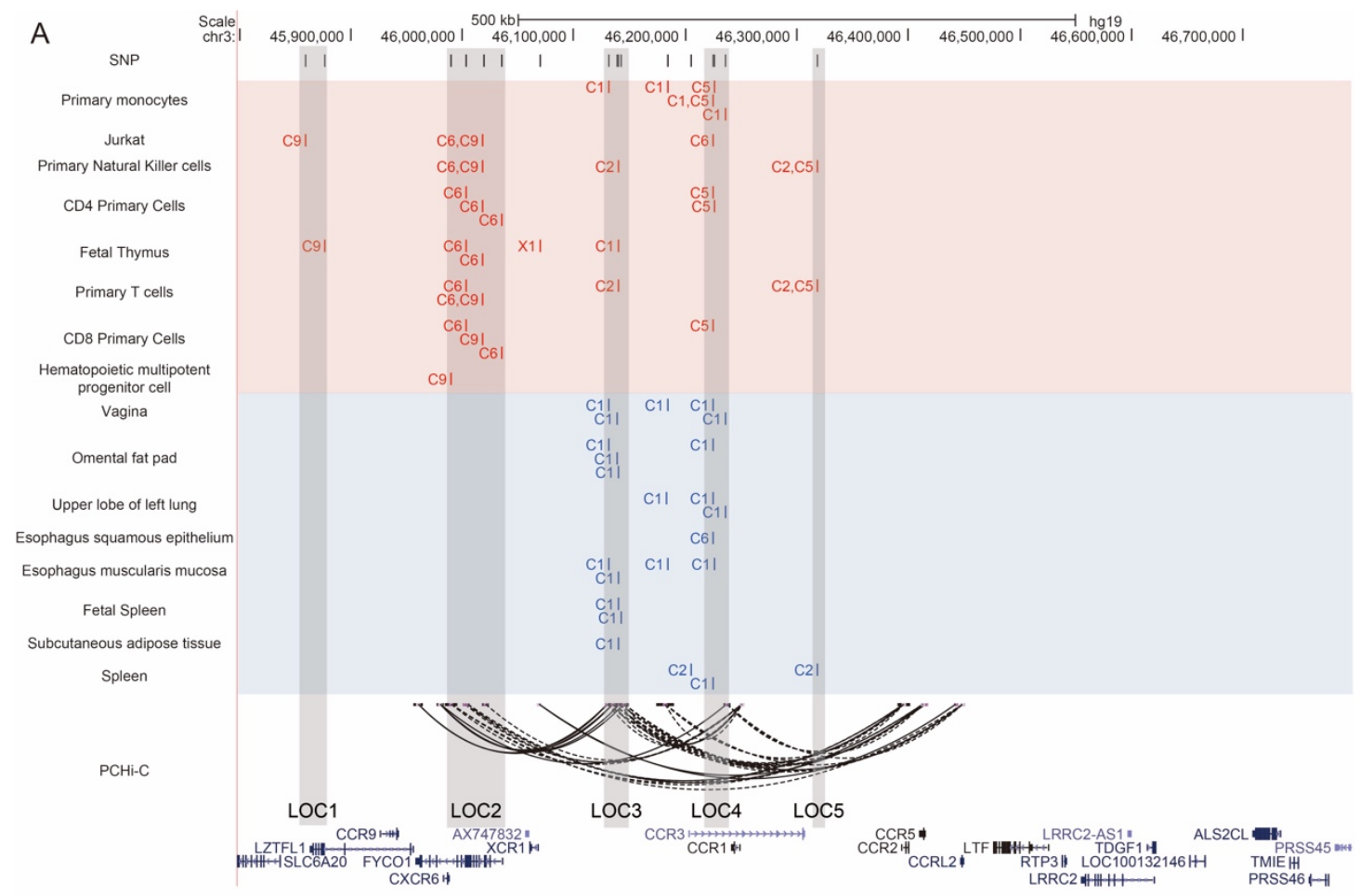

B

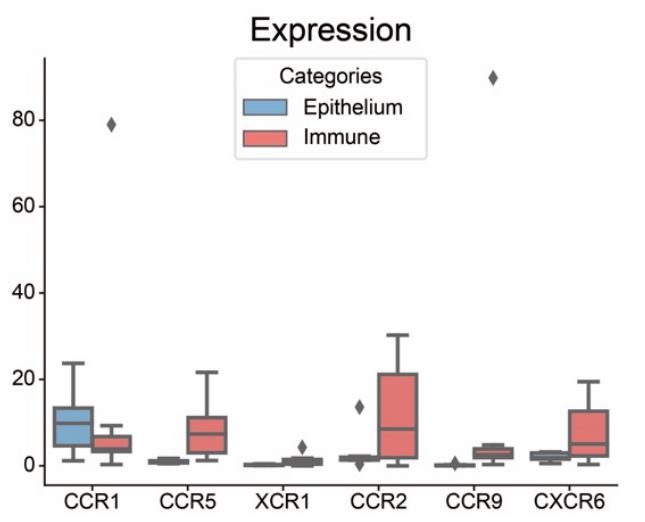

C

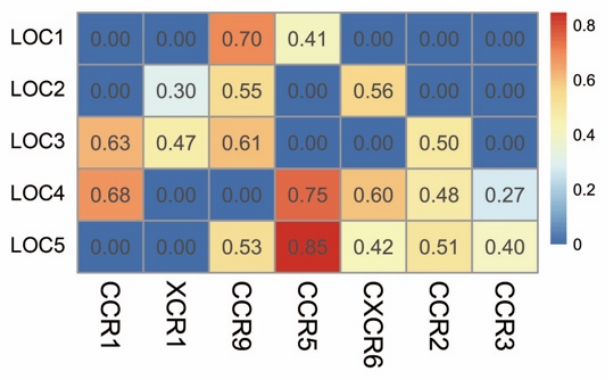

Figure 6. (A). SNPs, REs and PCHi-C loop associated with chemokine receptors. The RE tracks consisted of 11 immune cell types and 9 epithelium cell types. PCHi-C loops were from blood samples. The labels around REs were indicator of TGs: C1-CCR1, C2-CCR2, C5-CCR5, C6-CXCR6, C9-CCR9, X1-XCR1. (B). Expression of chemokine receptors in immune and immune cells. (C). Correlations between accessibility of REs and expression of chemokine receptors.

\section{Discussion}

The COVID-19 pandemic has influenced human's life all over the world for two years. Scientists made their efforts to gain more understanding of pathology of COVID-19 and find effective cure. GWAS is a powerful tool to discover suspicious loci for interesting traits. By profiling phenotype and genotype of COVID-19 infected people, the "COVID-19 host genetics initiative" had conducted GWAS analysis with more and more power ${ }^{34}$. Through these GWAS, many risk loci that may be associated with COVID-19 infection and severity were found. Following these discoveries, we used regulatory network atlas to interpret these risk loci and sought to gain biological insights of COVID19. 
First, we found that COVID-19 severity associated SNPs were mainly enriched in two types of human contexts: immune cells and epithelium cells. We used another GWAS of COVID-19 severity with larger cohort (https://www.covid19hg.org/results/r6/) to show that the association with immune cells and epithelium cells were quite reproducible (Figure S1). We then constructed the COVID-19 severity associated regulatory network in the two major contexts. And further analysis revealed regulatory structure of COVID-19 associated regulatory networks: the four regulatory clusters and two classes of upstream TFs. The regulatory structure showed conservation and divergence between two of cell types. Finally, we focused on two important regulatory clusters (OAS cluster and chemokine receptor cluster) and revealed the causal genes and differential regulation between two cell types.

Several factors hindered the post-GWAS analysis of COVID-19. First of all, one more powerful GWAS with larger cohort is in need. In this paper, the GWAS only detected 542 SNPs that significantly associated with COVID-19's symptoms, which is not comparative to normal phenotype, such as height or BMI. Some efforts have been made to improve the power of GWAS. For example, the most recent GWAS conducted by "COVID-19 host genetics initiative" has included more than 8,000 very severe respiratory confirmed cases into analysis. On the other hand, a more comprehensive regulatory network atlas is promising for better understanding of COVID19 severity. Although our current regulatory network atlas has covered many tissues, it is still far from complete. As the symptoms of COVID-19 are involved with many organs, a more comprehensive regulatory network atlas, even at cell type level, will interpret more genetic variants associated with COVID-19.

\section{Methods}

Construction of regulatory network from paired expression and chromatin accessibility data We utilized PECA2 to infer genome-wide and context-specific regulatory networks based on gene expression and chromatin accessibility data in that context ${ }^{13}$. Given paired RNA-seq and ATAC-seq data for a sample, PECA2 hypothesized that TF regulated the downstream TG by binding at REs. The regulatory strength of a transcription factor (TF) on a target gene (TG) was quantified by the trans-regulation score, which was calculated by integrating information from multiple regulatory elements (REs) that may mediate the activity of the TF to regulate the TG. A prior TF-TG correlation across external public data from ENCODE database was included in the trans-regulation score definition to distinguish the TFs sharing the same binding motif (i.e., TFs from the same family).

We collected paired expression and chromatin accessibility data of 76 human tissues or cell lines (Table S2) and applied PECA2 to these data to obtain regulatory networks of 76 human contexts. Furthermore, we recently constructed high-quality regulatory network of cranial neural crest ${ }^{15}$ and we included it into our analysis to form regulatory network atlas of 77 human contexts.

\section{Fold enrichment score of SNPs in given region set}

Given a group of SNPs and a RE set, we defined the fold enrichment (FE) score as follows,

$$
F E=\frac{P_{r} / L_{r}}{P / L}
$$


Where $P_{r}$ was the number of SNPs in REs. $L_{r}$ was the length of the REs. $P$ was the total number of SNPs. $L$ was the genome length.

We calculated the $F E$ score of 542 SNPs of COVID-19 severity in RE sets of 77 human contexts and used a criterion of $F E \geq 3$ to find 21 contexts that associated with COVID-19 severity.

\section{Extraction of SNPs associated regulatory network in given tissue and two categories}

Given a tissue, we check every RE in its regulatory network, if there was at least one SNP that was located in this RE, we extracted this RE and its upstream TFs and downstream TGs. We linked the SNPs in RE and this RE with edges and the SNPs, REs, TFs, TGs formed a SNP-associated regulatory network in a given tissue.

For 11 tissues of immune cells, we union the SNPs, REs, TFs, TGs. We assigned an edge to SNP$\mathrm{RE}$ if it existed in at least one tissue's SNP associated regulatory network, assigned an edge to TF$\mathrm{RE}$ if it existed in at least one tissue's SNP associated regulatory network, assigned an edge to RETG if it existed in at least one tissue's SNP associated regulatory network. After the union and edge assignment, we constructed a regulatory network of immune cells. The regulatory network of epithelium cells was constructed with the same procedure.

\section{Data availability}

The GWAS summary statistics of COVID-19 severity ("A2_ALL" study) was download at https://www.covid19hg.org/results/r4/. The constructed regulatory network atlas was freely available at https://github.com/AMSSwanglab. The eQTL dataset was download at the GTEx portal https://www.gtexportal.org/home/datasets. The collected paired expression and chromatin accessibility data was summarized in Table S1 and Table S2.

\section{Declaration of interests}

The authors declare no competing interests.

\section{Acknowledgements}

We acknowledge funding from the Strategic Priority Research Program of the Chinese Academy of Sciences (XDPB17), National Key Research and Development Program of China (2020YFA0712402), and the National Natural Science Foundation of China (grants 11688101,12025107, 11871463, 61621003). The Genotype-Tissue Expression (GTEx) Project was supported by the Common Fund of the Office of the Director of the National Institutes of Health, and by NCI, NHGRI, NHLBI, NIDA, NIMH, and NINDS. The data used for the analyses described in this manuscript were obtained fromthe GTEx Portal under dbGaP accession number phs000424.v8.p2 on 12/09/2020.

$\mathrm{T}$

\section{Reference}

1. Severe Covid, G.G. et al. Genomewide Association Study of Severe Covid-19 with Respiratory Failure. N Engl J Med 383, 1522-1534 (2020). 
2. Pairo-Castineira, E. et al. Genetic mechanisms of critical illness in Covid-19. Nature (2020).

3. Initiative, C.-H.G. The COVID-19 Host Genetics Initiative, a global initiative to elucidate the role of host genetic factors in susceptibility and severity of the SARS-CoV-2 virus pandemic. Eur J Hum Genet 28, 715-718 (2020).

4. Kumar, V., Wijmenga, C. \& Withoff, S. From genome-wide association studies to disease mechanisms: celiac disease as a model for autoimmune diseases. Seminars in Immunopathology 34, 567-580 (2012).

5. Smemo, S. et al. Obesity-associated variants within FTO form long-range functional connections with IRX3. Nature 507, 371-+ (2014).

6. Claussnitzer, M. et al. FTO Obesity Variant Circuitry and Adipocyte Browning in Humans. New England Journal of Medicine 373, 895-907 (2015).

7. Andersson, R. et al. An atlas of active enhancers across human cell types and tissues. Nature 507, 455-+ (2014).

8. Watanabe, K., Taskesen, E., van Bochoven, A. \& Posthuma, D. Functional mapping and annotation of genetic associations with FUMA. Nat Commun 8, 1826 (2017).

9. McLean, C.Y. et al. GREAT improves functional interpretation of cis-regulatory regions. Nat Biotechnol 28, 495-501 (2010).

10. Zhu, Z. et al. Integration of summary data from GWAS and eQTL studies predicts complex trait gene targets. Nat Genet 48, 481-7 (2016).

11. He, X. et al. Sherlock: detecting gene-disease associations by matching patterns of expression QTL and GWAS. Am J Hum Genet 92, 667-80 (2013).

12. Duren, Z., Chen, X., Jiang, R., Wang, Y. \& Wong, W.H. Modeling gene regulation from paired expression and chromatin accessibility data. Proc Natl Acad Sci U S A 114, E4914-E4923 (2017).

13. Duren, Z., Chen, X., Xin, J., Wang, Y. \& Wong, W.H. Time course regulatory analysis based on paired expression and chromatin accessibility data. Genome Res 30, 622-634 (2020).

14. Xin, J. et al. Chromatin accessibility landscape and regulatory network of high-altitude hypoxia adaptation. Nat Commun 11, 4928 (2020).

15. Feng, Z.Y. et al. hReg-CNCC reconstructs a regulatory network in human cranial neural crest cells and annotates variants in a developmental context. Communications Biology 4(2021).

16. Schaller, M.A., Kallal, L.E. \& Lukacs, N.W. A key role for CC chemokine receptor 1 in T-cellmediated respiratory inflammation. Am J Pathol 172, 386-94 (2008).

17. Kinoshita, S., Akira, S. \& Kishimoto, T. A member of the C/EBP family, NF-IL6 beta, forms a heterodimer and transcriptionally synergizes with NF-IL6. Proc Natl Acad Sci U S A 89, 14736 (1992).

18. Chinery, R., Brockman, J.A., Dransfield, D.T. \& Coffey, R.J. Antioxidant-induced nuclear translocation of CCAAT/enhancer-binding protein beta. A critical role for protein kinase Amediated phosphorylation of Ser299. J Biol Chem 272, 30356-61 (1997).

19. Roy, S.K. et al. MEKK1 plays a critical role in activating the transcription factor C/EBP-betadependent gene expression in response to IFN-gamma. Proc Natl Acad Sci U S A 99, 7945-50 (2002).

20. Pless, O. et al. G9a-mediated lysine methylation alters the function of CCAAT/enhancerbinding protein-beta. J Biol Chem 283, 26357-63 (2008).

21. Wang, S.Y. et al. Characterization of the relationship between FLI1 and immune infiltrate level in tumour immune microenvironment for breast cancer. Journal of Cellular and Molecular 
Medicine 24, 5501-5514 (2020).

22. Burgner, D., Jamieson, S.E. \& Blackwell, J.M. Genetic susceptibility to infectious diseases: big is beautiful, but will bigger be even better? Lancet Infectious Diseases 6, 653-663 (2006).

23. Noguchi, S. et al. Differential effects of a common splice site polymorphism on the generation of OAS1 variants in human bronchial epithelial cells. Hum Immunol 74, 395-401 (2013).

24. Cassel, T.N. \& Nord, M. C/EBP transcription factors in the lung epithelium. Am J Physiol Lung Cell Mol Physiol 285, L773-81 (2003).

25. Kabbout, M. et al. ETS2 mediated tumor suppressive function and MET oncogene inhibition in human non-small cell lung cancer. Clin Cancer Res 19, 3383-95 (2013).

26. Staal, F.J. \& Clevers, H. Tcf/Lef transcription factors during T-cell development: unique and overlapping functions. Hematol J 1, 3-6 (2000).

27. Chen, Z. et al. TCF-1-Centered Transcriptional Network Drives an Effector versus Exhausted CD8 T Cell-Fate Decision. Immunity 51, 840-855 e5 (2019).

28. Tamura, T., Yanai, H., Savitsky, D. \& Taniguchi, T. The IRF family transcription factors in immunity and oncogenesis. Annu Rev Immunol 26, 535-84 (2008).

29. Jiao, X. et al. Recent Advances Targeting CCR5 for Cancer and Its Role in Immuno-Oncology. Cancer Res 79, 4801-4807 (2019).

30. Sengez, B. et al. The Transcription Factor Elf3 Is Essential for a Successful Mesenchymal to Epithelial Transition. Cells 8(2019).

31. Deppermann, C. et al. Gray platelet syndrome and defective thrombo-inflammation in Nbeal2deficient mice. J Clin Invest (2013).

32. Mozzi, A. et al. OASes and STING: adaptive evolution in concert. Genome Biol Evol 7, 101632 (2015).

33. Javierre, B.M. et al. Lineage-Specific Genome Architecture Links Enhancers and Non-coding Disease Variants to Target Gene Promoters. Cell 167, 1369-1384 e19 (2016).

34. Pairo-Castineira, E. et al. Genetic mechanisms of critical illness in COVID-19. Nature 591, 9298 (2021). 